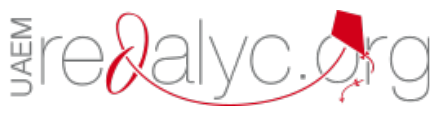

Centro Sur

ISSN: 2600-5743

compasacademico@icloud.com

Grupo Compás

Ecuador

El material didáctico potencia la enseñanza de los docentes en formación participantes de la estrategia itinerante Aula Móvil

Ramírez, Paola Andrea; Cabezas Guzmán, Vanesa Andrea; Rodríguez, Angie Lizeth; Acero, Mary Luz El material didáctico potencia la enseñanza de los docentes en formación participantes de la estrategia itinerante Aula Móvil

Centro Sur, vol. 3, núm. 2, 2019

Grupo Compás, Ecuador

Disponible en: http://www.redalyc.org/articulo.oa?id=588861691005

Esta obra está bajo una Licencia Creative Commons Atribución-NoComercial-SinDerivar 4.0 Internacional. 


\section{El material didáctico potencia la enseñanza de los docentes en formación participantes de la estrategia itinerante Aula Móvil}

The teaching material enhances the teaching of teachers in training participants of the mobile classroom itinerant strategy

Paola Andrea Ramírez pramirezra1@uniminuto.edu.co

Colombia

http://orcid.org/0000-0002-6845-8224

Vanesa Andrea Cabezas Guzmán vcabezasguz@uniminuto.edu.co

Colombia

http://orcid.org/0000-0003-4302-2050

Angie Lizeth Rodríguez ariodrig@uniminuto.edu.co

Colombia

Centro Sur, vol. 3, núm. 2, 2019

Grupo Compás, Ecuador

Recepción: 06 Noviembre 2018 Aprobación: 24 Diciembre 2018

Redalyc: http://www.redalyc.org/ articulo.oa?id $=588861691005$
Resumen: El concepto de material didáctico ha sufrido una serie de transformaciones a través del tiempo, comenzando porque atiende a distintos nombres tales como recursos, medios y ayudas pedagógicas, sin embargo se llega a la conclusión que todos los anteriores cumplen una función, son herramientas que facilitan y apoyan el proceso de enseñanza aprendizaje tanto del docente como del estudiante, Es por esto que el propósito de la investigación es dar a conocer como el material didáctico influye en la enseñanza de los docentes en formación brindada a las infancias participantes de la estrategia itinerante Aula Móvil, definida como aquella que fortalece las dimensiones del ser a través de una educación no formal que visita las 6 zonas más vulnerables del municipio de Soacha y sus 2 corregimientos. Se desarrollaron los objetivos a través del paradigma interpretativo de corte cualitativo, que permite acercarse al conocimiento de una problemática, entenderla, y comprenderla, para luego realizar un análisis e interpretación del objeto de estudio, por medio de algunas entrevistas semi - estructuradas a algunos docentes en formación, padres de familia y niños participantes de Aula Móvil; adicional a ellos se pretende mostrar como de esté depende un buen ejercicio pedagógico, que genere experiencias significativas Asimismo, se busca exponer algunas de las características básicas que se necesitan para trabajar con un excelente material didáctico, que consigo lleva una intencionalidad educativa, más específicamente se quiere demostrar que en muchas ocasiones no es la falta de material sino de utilidad y finalidad del mismo dentro de los procesos de enseñanza aprendizaje. Se espera con esta investigación reconocer el valor que cumple el material didáctico dentro del proceso educativo y sus principales aportes en cualquier tipo de formación.

Palabras clave: Material didáctico, aprendizaje significativo, infancias, itinerante, Aula Móvil.

Abstract: The concept of didactic material has undergone a series of transformations over time, starting because it attends to different names such as resources, means and 
pedagogical aids, however it is concluded that all the previous ones fulfill a function, they are tools that facilitate and support the teaching-learning process of both the teacher and the student, This is why the purpose of the research is to show how the teaching material influences the teaching of teachers in training provided to the participating childhoods of the itinerant classroom strategy Mobile, defined as one that strengthens the dimensions of being through a non-formal education that visits the 6 most vulnerable areas of the municipality of Soacha and its 2 districts. The objectives were developed through the qualitative interpretative paradigm, which allows us to approach the knowledge of a problem, understand it, and understand it, and then perform an analysis and interpretation of the object of study, through some semistructured interviews with some teachers in training, parents and children participating in the Mobile Classroom; In addition to them, it is intended to show how a good pedagogical exercise depends on this, which generates significant experiences. Likewise, it seeks to expose some of the basic characteristics that are needed to work with an excellent didactic material, which carries with it an educational intentionality, more specifically He wants to demonstrate that in many occasions it is not the lack of material but of its usefulness and purpose within the teaching-learning processes. It is expected with this research to recognize the value of the teaching material in the educational process and its main contributions in any type of training.

Keywords: Teaching Materials, Significant Learning, Childhoods, Itinerant.

\section{INTRODUCCIÓN}

El presente trabajo de investigación pretende mostrar la importancia del material didáctico para la implementación de una práctica idónea en las docentes en formación en la estrategia itinerante Aula Móvil. De igual manera, el propósito fue fortalecer la elaboración de material que apoyará el proceso de enseñanza aprendizaje teniendo en cuenta características de calidad, color, resistencia, estética, y versatilidad de acuerdo a la edad de la población infantil y los temas a trabajar en los ambientes de aprendizaje que componen Aula Móvil.

El material didáctico se vuelve fundamental como estímulo a la hora de presentarlo a los niños en cada una de las actividades lúdico-pedagógicas en los diferentes ambientes de aprendizaje. Aula Móvil es una estrategia de educación no formal y en el municipio son insuficientes este tipo de instituciones que ofrezcan cobertura total a las infancias. Es por esto que, el material didáctico y recursos para la música, artes, deportes son limitados. Sin embargo es indispensable para que los niños y niñas aprendan de manera dinámica, creativa y fuera del contexto escolar cotidiano del aula regular.

Teniendo en cuenta lo anterior, se plantea como objetivo general: Analizar los recursos didácticos utilizados por los docentes en formación en la enseñanza a las infancias dentro de la estrategia itinerante Aula Móvil. Atendiendo las categorías, aspectos y características que debe tener un material didáctico planteado por diversos autores para que sea pertinente y adecuado en la enseñanza y permita el desarrollo integral de los niños y las niñas.

Para el desarrollo del proyecto y dar cumplimiento al objetivo se hizo necesario revisar en un primer momento referentes teóricos sobre los recursos didácticos con el fin de analizar la importancia de éstos en el proceso de enseñanza aprendizaje en la infancia. De la misma 
forma, fue fundamental analizar el material de los diferentes ambientes de aprendizaje que componen el Aula Móvil a través de algunos instrumentos como fichas de observación y entrevistas con el propósito de conocer qué material estaban utilizando, fortalezas y debilidades de éste para finalmente diseñar unas orientaciones en su elaboración e implementación.

Aula Móvil

Con el creciente desplazamiento de personas que llegan al Municipio de Soacha se presenta una superpoblación que la administración municipal no alcanza a atender y brindar las condiciones básicas necesarias para vivir en forma armónica. Es por esto que, se presentan problemáticas a nivel social, cultural y político. En cuanto a la educación formal, falta cobertura total y la educación no formal es insuficiente para los niños, jóvenes y adultos de la región. Surge entonces la necesidad de apoyar a las infancias en actividades lúdico-pedagógicas inclusivas que complemente la educación regular, aprovechen el tiempo libre y se diviertan.

Aula Móvil recorre las seis comunas y dos corregimientos del municipio en donde se trabaja la fundamentación teórica, las prácticas pedagógicas y la investigación. Asimismo, permite como campo de práctica desarrollar y fortalecer las habilidades y competencias de las estudiantes de la Licenciatura, de igual modo la participación directa con las comunas genera una mirada a los diferentes contextos a donde se llega reconociendo diferentes factores políticos, económicos y culturales.

En una primera mirada Aula Móvil es un escenario netamente pedagógico el cual brinda una educación no formal que complementa el sistema escolar a niños y niñas del Municipio de Soacha, es así como a través de las actividades lúdico pedagógicas, planeadas por los estudiantes en formación potencian y desarrollan las dimensiones del ser humano (ético, corporal, comunicativa, estética, espiritual, socio- afectiva y cognitiva).

Esta mirada de investigación nace del trabajo del Semillero Primera Infancia y del Proyecto de Investigación: Pedagogía al contexto Aula Móvil donde se trabaja de manera articulada los docentes en formación que realizan su práctica profesional y los estudiantes del semillero de investigación que observan este trabajo y lo convierten en análisis y reflexión de ese quehacer académico para posteriormente realizar sus opciones de grado, es decir, se articula la práctica con la investigación y la proyección social en beneficio de la comunidad.

En el desarrollo del trabajo en Aula Móvil se hace una convocatoria (los docentes en formación), se diseñan unas planeaciones con el material didáctico correspondiente que permita la implementación de las actividades lúdico pedagógicas teniendo en cuenta las necesidades de la población a la que se dirigen, para ello, se realiza una visita con anterioridad al presidente de la junta de acción comunal donde se va a trabajar con el fin de organizar los horarios, espacios y necesidades.

Aula móvil toma las orientaciones pedagógicas planteadas por María Montessori con su método Pedagogía Científica, el cual consiste en la implementación de material didáctico específico para ejercitar las 
diferentes áreas del desarrollo con el fin de generar ambientes ricos en la libertad y el amor por los niños, "si la pedagogía ha de surgir del estudio individual de la escuela, el estudio ha de provenir de la observación de los niños libres, de los niños estudiados y vigilados, pero no oprimidos" (Trilla, 2007).

El material didáctico se compone de tres elementos fundamentales: la función motora, el desarrollo sensorial y el lenguaje. Montessori (1967) considera esenciales para el aprendizaje, la práctica, la imitación, la repetición, la ordenación y la clasificación. Elementos fundamentales que componen cada planeación.

Otro de los autores que apoya el trabajo de Aula Móvil es Ausubel (1968) quien presenta el aprendizaje significativo como aquel que relaciona los conocimientos previos con los conocimientos nuevos mediados por un docente o guía. Sin embargo, más allá de relacionar conceptos, el autor afirma que el aprendizaje realmente se genera cuando el estudiante es capaz de poner en práctica el conocimiento para resolver diferentes situaciones de su vida diaria.

De igual manera, Aula Móvil como sitio de práctica cumple con aspectos de la educación regular como: la utilización de un modelo pedagógico, el uso de material didáctico y la evaluación. Lo que la diferencia de la educación formal es su movilidad, el que sea itinerante, lo que permite identificar las necesidades en zonas apartadas que se encuentran en condiciones de pobreza y marginalidad. Asimismo, la articulación de la docencia (práctica) con la investigación formando docentes investigadores, críticos y reflexivos; que proponen y construyan proyectos que beneficien y transformen las problemáticas observadas en cada una de las comunidades.

A continuación se describe de manera breve cómo se desenvuelve aula móvil en las diferentes comunas.

Las salidas de campo con Aula Móvil se realizan los sábados a las diferentes comunas y corregimientos con una previa convocatoria de los niños de ese lugar, de igual modo que con el proyecto educativo claro, con planeaciones, material didáctico y recursos necesarios para el desarrollo de estas actividades. Se trabaja por ambientes de aprendizaje: rincón del cuento, matemático, pintura, creativo, corporal, Comunicativo, lógico y de experimentos los cuales permiten que el aprendizaje sea dinámico y divertido. Se termina con una dinámica de cierra. De igual manera, se evalúa el trabajo docente con los estudiantes en formación: cómo se llevó a cabo la jornada, aspectos positivos y por mejorar en cuanto a organización, planeación, uso pertinente del material didáctico, manejo a la población participante actitudes y aptitudes de estos.

Material didáctico

El material didáctico en educación infantil es fundamental por su alta influencia en el proceso de enseñanza- aprendizaje en los niños y niñas de 0 a 6 años, por ello la importancia de investigar cómo se utilizan y cuáles son las características que deben tener para contribuir a un mejor aprendizaje y con ello a una educación de calidad. 
El material didáctico cumple la función de apoyar el proceso de enseñanza aprendizaje, sin embargo muchos de estos materiales no son diseñados específicamente para un fin pedagógico, no obstante gracias a la creatividad, innovación y derroche de imaginación los docentes, transforman su contenido para suplir las necesidades de los estudiantes con el fin de alcanzar las competencias que exige la nueva sociedad, cuando se habla de material y recurso, se hace referencia a todo un conjunto de herramientas y elementos con los cuales interactúa el estudiante para comprender su entorno, así lo afirman Ameijeiras (2009),

Todo los elementos que rodean al niño tienen como primera función la de ser elemento mediador entre su conocimiento y la realidad que lo rodea, es como la puerta a través del cual el niño empezara a descubrir, a explorar, todo su entorno (p.330).

De igual manera, se resalta la importancia de seleccionar el material didáctico adecuado para cada planeación con el cual se trabajan conocimientos específicos con diferentes clases de materiales, tal como lo plantea (Marqués, 2000),

“¿Los materiales pueden clasificarse, sobre todo, en dos tipos según el fin por el cual han sido elaborados, de esta manera nos encontramos con los medios didácticos, que engloban a todo tipo de producción o material elaborado con una finalidad educativa; y los recursos educativos, donde se aglutina a todo ese material cuya confección no ha sido ideada para el ámbito educativo, pero si se utiliza con fines didácticos (p. 330).

Igualmente, Montessori (1967) tuvo experiencias con niños de escasos recursos, sus ideas principales se basaban en la asombrosa capacidad de los niños de aprender y desenvolverse en diferentes aspectos. Además, para ella lo más importante era la libertad de los niños puesto que esto generaba grandes ventajas, una de ellas era que podría llegar a crecer como un adulto capaz de hacerle frente a cualquier problema que se le presentase en la vida.

El impacto de la metodología Montessori fue tan grande que renovó todos los métodos pedagógicos del siglo XX siendo está una herramienta que apoya el proceso mental del niño, ayudándolo a entender y aprender de manera individual y significativa él mismo, por medio de la asociación de términos abstractos con experiencias sensoriales concretas donde se puede evidenciar el afianzamiento del conocimiento de manera dinámica, no solamente memorizada.

De igual manera, esta autora plantea que el material didáctico al ser una experiencia concreta, hace que el trabajo no sea únicamente de aprendizaje, sino también una inclusión de conocimientos previos y construidos a base de estos recursos didácticos, al respecto afirma "permite al niño apropiarse de unas habilidades de tipo lógico y clasificatorio, esenciales para el desarrollo de operaciones mentales de mayor complejidad".

Montessori propone unas características del material didáctico, tales como: tamaños, pesos, colores, formas, texturas, ductilidades, sonidos musicales, olores, esto en cuanto a su cualidad física, pero también expone el Auto-corrector, es decir, que el material usado le permita al niño darse cuenta de sus propios errores en el momento que realiza la actividad, 
como por ejemplo: los encajes de madera, fichas de rompecabezas etc. Otra de las características es la estética, hace referencia a que el material debe ser atractivo, llamativo, pintado con colores muy brillantes, material de calidad.

Este material didáctico pueden ser utilizados de manera individual o grupal, de esta forma se propicia el fortalecimiento de la comunicación y el intercambio de ideas, para que esto suceda el docente debe tener en cuenta cuatro valores fundamentales que permiten que la enseñanza brindada tanto en aula como fuera de ella, o en este caso, según el énfasis de esta investigación itinerante, que sea pertinente y adecuado.

Estos cuatro valores fundamentales para el desarrollo del material didáctico son: valor funcional, experimental, de estructuración y de relación. A partir de estos cuatro valores el material no es únicamente estructural y experimental, ya que proporciona a la infancia la posibilidad de experimentar y desarrollar los sentidos de acuerdo a su interés.

Importancia del material didáctico

El material didáctico influye en el rendimiento académico, la carencia de los recursos didácticos, la calidad o incluso también la cantidad del material desde lo más simples como pueden ser las pinturas, cartulinas, colores, tijeras hasta los más sofisticados como son los equipos audiovisuales y otros dispositivos entorpecen o facilitan la enseñanza.

Como el propósito de la investigación es dar a conocer cómo el material didáctico influye en la enseñanza de los docentes en formación brindada a las infancias participantes de la estrategia itinerante Aula Móvil en cada uno de los ambientes de aprendizaje, es importante reconocer el modelo utilizados por los docentes: un método de enseñanza tradicional donde los estudiantes son sujetos pasivos con aprendizajes memorísticos carentes de manipulación y utilización de materiales didácticos, o por el contrario, donde se le permite al estudiante construir su propio conocimiento, con participación activa que lo lleve a construir aprendizajes significativos Comenios (1652).

Proceso de enseñanza

La didáctica es el proceso de enseñanza-aprendizaje, la forma como enseña el docente e igualmente, la manera en que el estudiante adquiere sus aprendizajes. De igual manera, ésta fortalece el trabajo dentro y fuera del aula, es un apoyo pedagógico que brinda estímulo positivo y facilita cualquier aprendizaje. Es importante que el ambiente que se forme o construya a través del material didáctico debe ser lo más significativo posible y coherente con la realidad del estudiante, tal como lo expone Nérici citado por Torres (2009),

"La didáctica es el estudio del conjunto de recursos técnicos que tienen por finalidad dirigir el aprendizaje del alumno, con el objeto de llevarle a alcanzar un estado de madurez que le permita encarar la realidad, de manera consciente, eficiente y responsable, para actuar en ella como ciudadano participante y responsable ( $\mathrm{p}: 12)$.

En el mismo sentido, la didáctica tiene varias intencionalidades pedagógicas busca que el conocimiento se construya de una manera creativa e innovadora para facilitar el proceso de enseñanza - aprendizaje. 
Además, permite al docente enriquecer su saber desde diferentes fuentes investigativas que lo obligan a estar en constante estudio para afrontar los nuevos retos de enseñar en este siglo, Federación de Enseñanza de (CC.OO,2009)

“... el empleo de recursos de enseñanza tiene un doble contenido: por un lado, mejorar el aprendizaje y por otro, crear condiciones para que profesores y alumnos interactúen dentro de un clima donde domina el ambiente con el fin de extraer del mismo los mejores resultados para su formación" (p: 2).

En el mismo sentido, todo material didáctico debe estar acompañado de una metodología la cual parte de una intencionalidad pedagógica, la transmisión de uno o varios saberes, a través de ellos no solo se facilita la transmisión del conocimiento, sino que se muestra la capacidad del docente para crear e innovar nuevos recursos didácticos, que suplan las nuevas necesidades que tienen los estudiantes, así lo plantea Henao (2012),

"el material didáctico favorece el proceso de aprendizaje en los estudiantes, gracias al contacto practico lúdico con elementos reales que activan el gusto por aprender, que estimulan el desarrollo de la memoria, la motricidad, fina y gruesa, la parte cognitiva, física entre otros, aspectos fundamentales en la evolución del sujeto" (pág. 105).

Clases de material didáctico

En la actualidad el material didáctico tiene como objetivo llevar al estudiante a investigar, descubrir y construir, para adquirir de manera dinámica un nuevo conocimiento, desde su experiencia y con una aproximación de la realidad.

De acuerdo a lo anterior, se plantean varios parámetros y características que debe cumplir este material desde un punto de vista pedagógico, comunicativo e informático. La importancia que tienen los procesos de innovación dentro de la enseñanza ha llevado a relacionarla con el uso de recursos físicos, teniendo en cuenta que estos hacen parte de la intermediación del acto de enseñar y la formación docente.

Existen diferentes clasificaciones de los recursos didácticos, sin embargo, pueden subdividirse en categorías siguiendo la teoría de Comenios (1657),

Convencionales: Los materiales en las instituciones, se basa en la enseñanza por medio de textos guías, libros y recursos físicos que permiten que el estudiante memorice el concepto y lo ejercite diariamente de manera escrita y tradicional. Audiovisuales: Es todo aquel contenido realizado con el fin de promover el uso de imágenes, facilita el proceso de enseñanza al llevar al estudiante la tecnología e ilustración por medio de su interés visual y auditivo. Como Tecnológicos: que integra la combinación de gráficos, textos, sonidos y videos, además le permite al estudiante desarrollar memoria, sistematizar y organizar la información.

Teniendo en cuenta las cuatro fases inducción, estructuración, consolidación y retroalimentación. Los materiales didácticos deben ser empleado como estrategia que permite y facilita el proceso de enseñanza 
del estudiante como el proceso de formación del docente, por lo que se bebe tener en cuenta (Comenios (1657),

La adecuación al ritmo de trabajo de los estudiantes, los buenos materiales tienen en cuenta las características psicoevolutivas de los estudiantes a los que va dirigido a partir de (desarrollo cognitivo, capacidades, intereses, necesidades...)

Estimular el desarrollo de habilidades meta cognitivas y estrategias de aprendizaje en los estudiantes, que les permitirán planificar, regular y evaluar su propia actividad de aprendizaje, fomentando la reflexión sobre su conocimiento y sobre los métodos que utilizan para realizar el proceso cerebral y organización del pensamiento. Ya que aprender significativamente implica modificar los propios esquemas de conocimiento, organizar, revisar, ampliar y enriquecer las estructuras cognitivas.

Los materiales de clase deben facilitar aprendizajes significativos y transferibles a otras situaciones mediante una continua actividad mental y experiencia de la realidad

La inclusión de los materiales didácticos en un determinado contexto educativo exige que el docente correspondiente tenga claro cuáles son las principales funciones que pueden desempeñar los medios didácticos en el proceso de enseñanza Comnios (1657).

La Enseñanza

La enseñanza está en relación a la transmisión de conocimientos e ideas, basado en diversos métodos teniendo como finalidad un aprendizaje por parte del sujeto. Ésta se define como la interacción que se da entre una o más personas en donde se transmiten diferentes conocimientos e ideas. Está se da en distintos contextos laborales, académicos e incluso personales, tal como lo expone Medina y Salvador (2003).

La enseñanza "Es comprendida cómo una actividad generadora de interacciones, promovedora de una inteligencia socio-afectiva y de actitudes singulares, a la vez que creadora de valores de colaboración y comunidad tolerante y de esfuerzo compartido. (p: 53).

Según lo anterior la enseñanza se da gracias a la comunicación dada en un contexto con diferentes fines informativos, por lo cual se requiere de la interacción y participación de una comunidad.

\section{MATERIALES Y MÉTODOS}

Para la presente investigación se tuvo en cuenta la población infantil niños y niñas entre el nacimiento a los 12 años, algunos padres de familia participantes de la estrategia itinerante Aula Móvil y finalmente los docentes en formación de la Licenciatura en Pedagogía Infantil de Práctica profesional.

La muestra con la que se trabajó fueron los docentes en formación de la Licenciatura, ya que el objetivo general planteado fue caracterizar el uso de material didáctico y cómo influye éste en la enseñanza de los docentes en formación que realizan su práctica profesional en este sitio de práctica itinerante. 
Para el desarrollo de la misma se tuvo en cuenta el paradigma interpretativo de carácter cualitativo, que consiste en la recolección de datos, análisis e interpretación del objeto de estudio. Se utilizaran entrevistas dirigidas a distintos agentes entre ellos docentes, padres de familia y niños con el fin de identificar cuál es su punto de vista frente a la importancia del material didáctico implementado en el proceso de enseñanza dentro de la estrategia.

De igual modo, se utilizaron fichas de observación para complementar la información, que permite al investigador conocer más detalladamente el objeto de estudio y así mismo lo que lo rodea. Estos instrumentos permiten observar, indagar y exponer qué importancia tiene la elaboración y diseño del material didáctico dentro del proceso de enseñanza teniendo en cuenta que está es itinerante y los docentes en formación lo deben cargar a las diferentes comunas y corregimientos.

La ponencia se desarrolla en el Municipio de Soacha, el cual tiene mayor porcentaje rural que urbano. Cuenta con seis comunas y dos corregimientos, históricamente se desarrolla bajo una nación indígena muisca quienes combinaron el "Sua" que significa sol y el "Cha" que significa Varón dando entonces la definición de Soacha como el municipio del varón del sol. De igual forma, cuenta con lugares y parques que encierran la cultura, fauna y flora del municipio como:

El parque Natural Chicaque miembro de la Unidad de Reservas Naturales, cuenta con 7 tipos de bosques y más de 100 especies de aves, este centro ecológico tiene dispuesto para los turistas dos zonas de camping con agua, comida, hostal cabañas y nidos (cabañas en los arboles) un plan perfecto para compartir en familia y conectarse con la Naturaleza.

Otro de los lugares de interés es el Salto del Tequendama, el cual es una catarata de 157 metros, es un sitio histórico de gran importancia ya que allí se encontraron los fósiles del hombre prehistórico más antiguo de Colombia, también se encuentra junto a él un antiguo hotel que en un pasado fue el paradero de aquellos que viajaban en tren y decidían hospedarse después de largos viajes. En la actualidad es una casa museo, visitada por varios turistas provenientes de distintos lugares del país.

Soacha presenta una serie de problemáticas que afectan la comunidad, como:

Desplazamiento diario de personas oprimidas que llegan desde diferentes puntos del país por diferentes causas como: pobreza e inseguridad, el transporte público es escaso, falta de cobertura en educación, en salud y falta de fuentes de empleo.

Aula Móvil se implementa como estrategia para desarrollar las prácticas profesionales de las y los estudiantes de la licenciatura, generando situaciones didácticas que permiten un acercamiento real del ejercicio docente permitiendo atender a población infantil que se encuentra en zonas de vulnerabilidad. Dicha educación no formal está acompañada de actividades pedagógicas, lúdicas y recreativas que potencian el desarrollo integral de los niños y niñas participes de Aula Móvil siendo este objeto de reflexión entre la teoría y la práctica. 
Es así como el Aula Móvil se compone de siete ambientes pedagógicos: Exploro mi cuerpo, juego y aprendo, Soacha mi territorio, Comunico y expreso lo que siento, En el mundo de las Tics, Viajando por el mundo mágico de la creatividad y padres con responsabilidad y amor, cada uno pertenece a una de las dimensiones del ser cognitiva, corporal, comunicativa, estética, socio- afectiva, ética y espiritual. En cada ambiente se encuentran actividades lúdicas pedagógicas relacionadas con temáticas alusivas a la dimensión acompañada de un material didáctico elaborado por los mismos estudiantes en formación.

La jornada de trabajo dentro del Aula Móvil se organiza de la siguiente manera: en el transcurso de la semana uno o dos de los estudiantes que lideran, se dirigen hacia la comuna donde llegara Aula Móvil. Allí se comunican con el presidente de la junta de acción comunal quien se encarga de gestionar los espacios y horarios para el desarrollo de la actividad, seguidamente se realiza la publicidad (convocatoria) del evento, está se realiza a través de carteleras que contienen toda la información del Aula Móvil, así como por medio de un pequeño perifoneo por todo el barrio.

Llegado el día sábado los estudiantes en formación se encuentran se desplazan al lugar donde se llevara a cabo la actividad comuna o corregimiento. Se realiza actividad de bienvenida, luego se rota por los diferentes ambientes de aprendizaje por grupos cada 30 minutos para disfrutar de las actividades preparadas. Para terminar, se realiza una dinámica de despedida y se les brinda un refrigerio.

Finalmente, se realiza la evaluación en donde se analizan la salida. El lugar fue adecuado, las actividades, el material didáctico. Es decir se revisa toda la parte de logística, desarrollo pedagógico, las habilidades de los docentes en formación con el grupo de niños y padres y se establecen los aspectos a mejorar.

\section{RESULTADOS}

Para la presentación de los datos y resultados obtenidos durante el proceso, se tuvo en cuenta, el análisis de entrevistas realizadas a docentes en formación, padres y niños participantes de Aula Móvil, para ello se hizo uso de las fichas de observación diligenciadas por las misma docentes, las entrevistas a docentes en formación, a padres de familia y niños participantes de Aula Móvil con el propósito analizar el material didáctico utilizado. 


\section{Análisis de entrevistas}



El autor 
Para la recolección de información del sentir de los docentes en formación en cuanto al uso y elaboración del material, se plantearon unas preguntas que recogen las opiniones e importancia que ellos le dan al material didáctico en el quehacer docente. A continuación se muestran estas preguntas con sus respuestas y su análisis.

¿Para usted que es material Didáctico?

¿En el proceso de enseñanza aprendizaje que valor le da al material didáctico? ¿por qué?

¿Usted elabora el material didáctico que usara, en las planeaciones en cada actividad?

¿Qué aspectos tiene en cuenta para la elaboración del material didáctico?

Teniendo en cuenta, la gráfica 1 , se puede observar frente al análisis de la primera categoría que los docentes en formación participes de Aula Móvil no tienen suficiente claridad frente a la importancia de la estética dentro del material didáctico, no basta solo con que apoye el proceso de enseñanza, sino que debe cumplir con unas características relevantes, tal como lo afirma Montessori:

"El material como todo lo que rodea al niño en el método Montessori, es atractivo, pintado de brillantes colores, sobrio, simple, elaborado con materias primas de gran calidad para suscitar su interés. Pero no basta con estética, al contrario debe ser susceptible de manipulación, debe prestarse a la actividad del alumno".

De la misma forma, se evidencia que los estudiantes en formación tienen clara la fundamentación teórica frente a lo que es el material didáctico, saben que se utiliza para apoyar el proceso de enseñanza aprendizaje, y que gracias a él se pueden generar grandes experiencias significativas, así como lo expone (Guerrero, 2009).

También consideramos materiales didácticos a aquellos materiales y equipos que nos ayudan a presentar y desarrollar los contenidos y a que los/as alumnos/as trabajen con ellos para la construcción de los aprendizajes significativos. Material didáctico es cualquier elemento que, en un contexto educativo determinado, es utilizado con una finalidad didáctica o para facilitar el desarrollo de las actividades formativas ( $\mathrm{p}: 12$ ).

Frente a la finalidad del material los docentes en formación tienden a confundirlo con su utilización, siendo estos términos diferentes, éste se utiliza con un propósito claro, es decir, existen cantidad de recursos didácticos y son los docentes quienes transforman esa utilización en algo innovador y llamativo según el objetivo propuesto una herramienta para facilitar la comunicación entre educador y alumno. Por tanto, son recursos que se utilizan con unos objetivos determinados en el contexto de una intervención.

Finalmente, en este análisis se concluyó con la pertinencia del material didáctico, gran parte de los docentes en formación demostraron claridad frente a la importancia de tener en cuenta el rango de edad al momento de elaborar el material didáctico dentro de la estrategias itinerante Aula Móvil, ya que allí participan niños de todas las edades y en diferentes contextos, sin embargo falta fortalecer el conocimiento frente a la 
coherencia con que se utiliza el material, como lo menciona Guerrero (2009),

"Los materiales facilitan la organización de las experiencias de aprendizaje, actuando como guías, no sólo en cuanto nos ponen en contacto con los contenidos, sino también en cuanto que requieren la realización de un trabajo con el propio medio; Adecuación al ritmo de trabajo de los/as alumnos/as. Los buenos materiales tienen en cuenta las características psicoevolutivas de los/as alumnos/as a los que van dirigidos (desarrollo cognitivo, capacidades, intereses, necesidades" ( $\mathrm{p}: 4$ ).

De la misma manera, para la recolección de información del sentir de los niños y niñas participantes de la estrategia itinerante Aula Móvil en cuanto a la innovación e interés frente al material se plantearon unas preguntas que recogen las opiniones e importancia que ellos le dan en el momento de participar en cada una de las actividades. A continuación se muestran estas preguntas con sus respuestas y su análisis.

1. ¿Te gustaron las actividades que hiciste el dia de hoy?.

- Si, me gustó las banderas y los numeros. (NP 1).

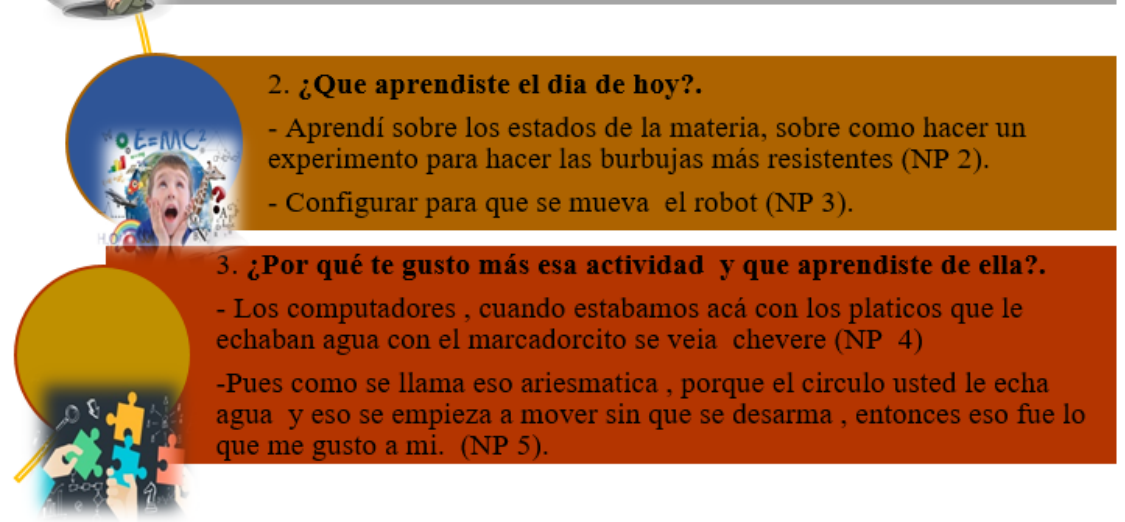

Grafico 2.

Entrevistas de los niños, niñas participantes en Aula Móvil.

Elaboración propia

Teniendo en cuenta el grafico 2, se puede observar que los niños muestran un alto grado de satisfacción con el material utilizado, pues lo consideran llamativo e innovador, les gustaron los colores, formas, tamaños etc. Así mismo, el material permite generar un aprendizaje significativo cuando se le da el debido uso y pertinencia frente a la temática que se quiere desarrollar.

De igual manera, el material utilizado permite identificar algunas emociones de los niños y niñas participantes de la estrategia Aula Móvil pues evidencias expresiones de alegría como "a mí me gusto", "se veía chévere" y "aprendí mucho" cuando se indaga por su apreciación de éste.

Finalmente, de este análisis se concluye la importancia de la elaboración del material didáctico, ya que como se observó anteriormente esté genera no solo un aprendizaje sino una serie emociones que le permiten al niño 
un acercamiento directo con el material lo cual lo lleva a la adquisición de nuevos conocimientos a través de algunas características como su color, forma, tamaño, innovación, que permiten en un primer momento llamar su atención y en un segundo momento generar un proceso de aprendizaje significativo.

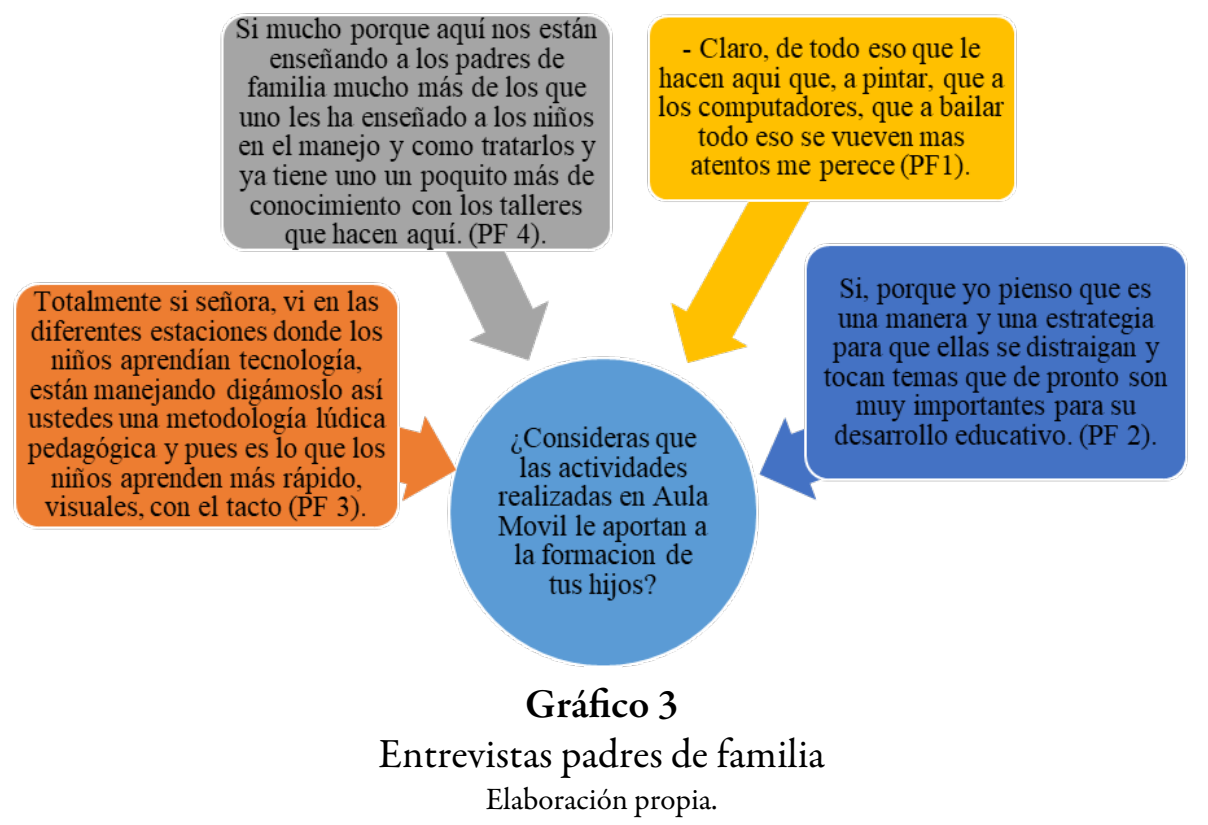

Para la recolección e interpretación del sentir de los padres de familia asistentes a la estrategia itinerante Aula Móvil en cuanto al material didáctico elaborado y utilizado por los docentes en formación dentro de cada uno de los ambientes de aprendizaje para el desarrollo de diferentes temáticas, surgen unas preguntas que permiten observar los distintos puntos de vista que ellos le dan a esté cuando sus hijos participan y disfrutan de este material.

Según la gráfica 3, se puede observar que los padres ven en la estrategia Aula Móvil una pedagogía totalmente diferente a la del sistema educativo formal, pues reafirman que allí se implementan actividades que llaman la atención de los niños permitiéndoles "De esta manera aprenden más rápido" (PF 3). Lo cual indica que dentro de los ambientes de aprendizaje se desarrollar de manera lúdica y creativa con apoyo de material didáctico las temáticas elegidas por cada docente en formación de la manera significativa

Por otra parte, los padres de familia tienen a deducir que actividades como el baile, la música, la pintura y el dibujo permiten que los niños y niñas sean más atentos, desarrollando así no solo su creatividad sino también la interacción de saberes con sus pares teniendo en cuenta que en este espacio pedagógico asisten niños de todas las comunas.

Finalmente, el uso de material didáctico permitió a los padres de familia ver la importancia de los mismos dentro del proceso de enseñanza y como depende de ellos generar y desarrollar procesos de aprendizaje significativo. Entendido como aquel que permite la puesta en práctica del conocimiento en diferentes escenarios de la vida cotidiana. 


\section{DISCUSIÓN}

Es necesario utilizar dentro de la estrategia itinerante Aula Móvil en las diferentes actividades pedagógicas material didáctico que cumpla con las características mínimas de durabilidad, accesibilidad, color, textura, tamaño, es decir que sea lo suficientemente llamativo y resistente para soportar cualquier contexto y adversidad.

Se pudo evidenciar que cuando existe material didáctico llamativo, novedoso, los niños desarrollan las competencias de manera más fácil lo cual deja claro que, aunque la estética de los materiales es importante lo es aún más su intencionalidad pedagógica.

Muchas de las respuestas de las entrevistas a padres de familia, arrojaron un alto grado de satisfacción frente a la implementación del proyecto Aula Móvil como lo manifiestan en sus respuestas mostradas anteriormente, proponiendo que ésta se realizara entre semana de manera continua, lo cual lleva a la profunda reflexión de cómo esta estrategia aprovecha el tiempo libre y enseña contenidos para la vida de manera lúdica.

Es importante que los docentes en formación tengan claridad y coherencia respecto a los objetivos que se plantean para cada actividad, así como la fundamentación teórica básica frente a conceptos como, material didáctico, planeaciones, propuesta pedagógica, didáctica, modelo, enfoque.

Dentro de cada ambiente se viven experiencias totalmente diferentes, lo cual lleva a una rigurosa y constante búsqueda de actividades y materiales que respondan a las nuevas necesidades, siendo estos innovadores, creativos, llamativos, pero especialmente productivos, que los docentes tenga claridad en la intencionalidad pedagógica.

Se pudo evidenciar que Aula Móvil carece de ayudas externas para su mantenimiento, como por ejemplo la alimentación corre por cuenta de los mismos estudiantes, los materiales son elaborados por ellos mismos, lo que ocasiona un gasto económico. Por tanto, se recomiendan las alianzas con el banco de alimentos o empresas que quiera apoyar el proyecto y que se les proporcionen recursos a los estudiantes para la elaboración de este material.

La participación en algunas comunas es menor que en otras, se evidencio que una de sus causas es la falta de elementos publicitarios que visualicen la imagen de lo que es Aula Móvil y como se desenvuelve, por esto sería importante que mercadeo y comunicaciones de la universidad se vincularan para publicitarla y hacer la convocatoria de los niños y niñas de los diferentes barrios y veredas del municipio.

El transporte es otro de los factores preocupantes, los docentes en formación se trasladan cada sábado en transporte público o a pie hacía el lugar donde se desarrollará la actividad, lo cual perjudica la conservación del material, puesto que al estarlo movilizando de manera brusca puede estropearse con facilidad.

La falta de acompañamiento constante del personal docente encargado de este proyecto, causa que en varias ocasiones que los estudiantes en formación cometan los mismos errores dentro de las actividades, no 
teniendo aun claridad de lo que se quiere lograr dentro de esta estrategia, recordando que es lo más cercano al ejercicio docente que se vive en la realidad (Acero, 2019).

\section{Referencias}

Ameijeiras, G. (2009). Los materiales didácticos en el aula. Temas para la educación. Revista digital para profesionales de la enseñanza, 5(1), 1-7

Cadavid Rojas, A.M., \& Calderón Palacio, I.C. (2004). Análisis del concepto enseñanza en las teorías curriculares de Lawrence Stenhouse y José Gimeno Sacristán. Revista Educación y Pedagogía 16(40), 143-152. Recuperado de http://bibliotecadigital.udea.edu.co/ bitstream/10495/3074/1/

CadavidAna_2004_AnalisisEnsenanzaCurriculares.pdf

Ausbel, D. (1968). Libro la teoría del aprendizaje significativo en la perspectiva de la psicología cognitiva. Nueva york, Estados Unidos: Octaedro.

CC.OO, F. (2009). La importancia de los recursos didácticos en la. Revista digitala para profesionales de la enseñanza, 4(1),1-6.

Comenios, J. (1652). Producción de material didáctico: los objetos de aprendizaje. Revista iberoamericana de educación a distancia, 11(1), 81-105

Comenios, J. a. (1957). Didactica magna. España: akal.

Graells, M. (2013). La manipulación de los materiales como recurso. Revista Universidad Católica San Antonio de Murcia. 9(especial).329-337.

Guerrero, A. (2009). Los materiales didácticos en el Aula. Temas para la Educación. Revista digital para profesionales de la enseñanza, 5(1),1-7.

Henao. (2012). Colección pedagogica formacion inicial de Docentes Centroamericanos de Educacion Primaria o Basica. Revista colombiana de ciencias sociales. $2(1)$

Huertas, F. (2009). Didáctica General. En H. T. Maldonado, Colección Pedagógica Formación Inicial de Docentes (pág. 165).Argentina: Coordinación Educativa Y Cultural Centroamericana.

Imideo G Nerice. (1973). Hacia una didáctica general dinámica. Revista Didáctica Esvin. 1(1),1-30.

Picardo Joao, O. (2004). Diccionario Enciclopedico de Ciencias de la Educacion. El Salvador: Centro de Investigación Educativa

Marqués, P. (2000). Impacto de las TIC en educación: Funciones y limitaciones. Universidad Autónoma de México, México

Montesorri . (1967). El material didáctico para la construcción de aprendizajes significativos. Revista Colombiana de Ciencias Sociales. 1(1), 101-108.

Montessori, M. (1967). Manual práctico del método. España: CEPE

Montessori, M. (1979). El método de la Pedagogía Cientifica. Madrid, España: Biblioteca Nueva.

Torres, H. G. (2009). Coordinación Educativa y cultural Centroamericana. Colección Pedagógica Formación Inicial de Docentres Centroamericanos de Educación Primaria o Básica. Didáctica General. Argentina: CECC/SICA

Trilla, J. C. (2007). El legado pedagógico del siglo XX parala escuela del siglo XXI. España: Grao. 